Committees. In particular, there may be imagined subjects which two or more Sections might be disposed to recommend to the Division for discussion, in lieu of arranging joint meetings of the Sections. The Committee of the Division, on its part, should be enabled to invite the advice of the sectional organizations on all appropriate questions. The Organizing Sectional Committees should be kept regularly informed of the activities of the Division.
The Committee should meet regularly throughout the year, at intervals determined by itself, and in particular it should hold a meeting at or near the time of the joint meetings of Organizing Sectional Committees in January, in order to assure the relations with the Sections referred to above.

The Committee should report to the Council as and when necessary, and annually through the Council to the General Committee.

\title{
Logic and Probability in Physics*
}

\author{
By Dr. C. G. Darwin, F.R.S.
}

$\mathrm{I}^{\mathrm{N}}$ choosing a theme for my address I was in some difficulty. The main subjects of present interest in physics, the nucleus of the atom, cosmic rays, and the phenomena at deep temperatures, are being dealt with in the discussions of our Section, so that they would be excluded even apart from the fact that I cannot speak on them with authority. It would have been possible for me to choose a narrower subject, but I could not feel that this would have possessed the general interest that such an occasion demands, and so with some trepidation I am venturing on an even wider theme and am going to touch on the philosophy of our subject. This is a dangerous thing to do for one who has never made more than the most superficial study of pure philosophy, but still I do not apologize for it, because it appears to me that recent scientific history has revealed a deep schism between the professional philosophers and the scientists, and this schism is worthy of examination.

General philosophy claims to be the critical subject which lays down for all of us what we may be allowed to think, and yet it has played no part whatever in the great revolutions of human thought of the present century-those connected with relativity and the quantum theory. It might have been expected that the scientists would have been constantly consulting the philosophers as to the legitimacy of their various speculations, but nothing of the kind has happened. Since no one can dispense with some sort of metaphysic, each scientist has made one for himself, and no doubt they contain many crudities, but it would seem that a deep interest in metaphysic is a disadvantage rather than an advantage to the physicist-at least I have the impression that those of my friends who are most inclined to

- From the presidential address to Section A (Mathematical and Physical Sciences) of the British Association, delivered at Cambridge on August 19. speculate on the ultimate things appear to be the ones whose scientific work is most hampered by doing so. Now I propose to risk a similar indiscretion. I want to embody in it the practical philosophy of a physicist, and I do not mean it as an attack on the pure philosophers, who are very reasonable people, only chargeable with the minor offence of not having made me want to read their books!

I had better begin by stating shortly the ideas I intend to discuss. There is a notable contrast between the way we think about things and the way we think we ought to think about them. We have set up as an ideal form of reasoning the formal logic which has held the field since the days of Aristotle. We rarely conform to this ideal, but instead we usually make use of arguments having no accurate axiomatic basis, which compel belief because of some large accumulation of favourable evidence. I intend to develop the idea that the old logic was devised for a world that was thought to have hard outlines, and that, now that the new mechanics has shown that the outlines are not hard, the method of reasoning must be changed. The key to the modification has already long been in our hands in the principle of probability, but whereas in the past constant attempts were made to fit this into the old system, the new mechanics suggests the possibility of a different synthesis. Though I hope this subject will be found interesting in itself, I would not have ventured to bring it forward if $I$ had not also a very practical purpose in doing so, and that is to urge that our mathematical education both at school and university has been gravely deficient in that it has put all the emphasis on matters susceptible of rigorous proof, while it has very completely neglected the equally important subjects of statistics and probability. I shall enter into these matters at the end of my address. 
If we examine various examples of theories and why we believe in them, we conclude that an axiomatic basis, of the kind demanded for the operations of formal logic, is too narrow for the understanding of the physical world. Something wider is needed. Now for more than a century there has been growing up the recognition that probability plays a part in much reasoning, and that there must exist a wider system of logic which has probability as one of its features. Attempts have been made, and are still being made, to bring probability back into the narrow fold of the old logic. It appears to me that these attempts are hopeless, but before approaching the question directly I want to develop an analogy which seems to me important. Like everyone else I feel the compelling power of the old logic, and I cannot feel how when we try to go beyond it we can get the same compulsive force. But on the other hand, I know of a case where our thoughts are driven in one direction by a force which seems to have the same psychological compulsion as does formal logic, and yet where the result is undoubtedly wrong.

To anyone who has thought at all seriously about the world, or at any rate to anyone who has made an elementary study of mechanics, I suppose there is nothing more absolute than the law of causality. By this I mean that the future is completely contained in the present. Passing over obvious examples where this is true, like the path of a projectile or the orbit of a planet, we may take an extreme case where we might expect our faith in the principle would be most severely tried. Take the typical case of chanciness, the tossing of a coin. We know that in a general way there is an even chance of heads or tails, even though we sometimes hear of gifted individuals with muscles so delicately adjusted that they can control the event. But in the ordinary way the tossing of a coin is complicated by being produced by a living organism, so let us simplify the problem by designing a catapult of some kind to project it. Which of us does not believe the coin would fall the same way every time if such a mechanism could be made with really complete precision? When the machine fails to make it do so, we say it is because there may have been a speck of dirt in the lubricant or something like that. In other words, we do not feel that the fall of the coin is determined by chance, but we regard the uncertainty we observe as due to our ignorance of all the detailed causes. Ignorance is a confession of incompetence, and so we regard the existence of chance as a blemish in our otherwise admirable characters. This feeling goes very deep, since we are prevented by it from having the complete control of our surroundings that we somehow think should be our due. We start prejudiced against probability and in favour of causality.

So much for what we feel about causality ; and about thirty years ago this feeling would have been regarded as a piece of inescapable reasoning, with the same kind of compelling power as a logical syllogism. We still have the feeling, but now we know it is wrong, and what is more, we know that it is wrong for a reason we never thought of. To understand this oversight we must review the recent history of atomic theory.

[There follows a review of the history of the quantum theory with special reference to the way in which it disposes of the difficulty of causality.]

The history of the quantum theory serves as an analogy to the deeper question of what is wrong with the old logical processes. Just as we used to feel the all-pervading compulsive force of causality, so we feel the all-pervading force of pure logic. Just as we felt that classical mechanics provided no room for anything beyond itself, so we feel that the old logic is the only admissible kind of reasoning. We knew that certain things led to the Old Quantum Theory and obstinately refused to fit into mechanies, and we know that the principle of probability can cover many things outside the old logic. Many men tried to force the quantum into the classical system, and many are still trying to bring probability within the fold of the old logic. I do not believe it can be done. This is not the occasion, nor have I the capacity, for a deep argument on the place of probability in logic, but one of the most convincing ways of seeing it may be found in the consideration of another branch of physical theory, the kinetic theory of gases.

The greatest contribution to the subject was that of Gibbs, who recognized that there had to be a big assumption somewhere and made it quite frankly and without attempt at justification. The works of Gibbs are not easy reading; in both his great works he attends to every detail with a particularity that is really rather tedious, whereas his basic ideas are thrown at the reader almost without explanation. The idea of a canonical ensemble is a really beautiful idea once you understand it, but where does it come from? An ensemble is an idea which will be unfamiliar to many, so I had better explain it. We want to know something about the behaviour of a complicated system composed of a great many parts ; say we want to know the pressure of the gas in some vessel. Gibbs considers a very large number of possible states of motion of the set of molecules, which have some character in common such as their total energy, but which are otherwise unrelated. Though each specimen of the 
motions is quite independent of all the others, he looks at them all together; this explains the word ensemble-I do not know why he had to take a French word-and makes the assumption that the pressure of the gas is correctly given by the average of all the specimens. The actual gas in the vessel at any instant is one of the specimens ; in its motion it passes into configurations corresponding to others, but only after a fantastically long time would it go through even a perceptible fraction of the whole ensemble. Gibbs is assuming that the behaviour of the actual gas will be determined by the average of the uncountable millions of specimens in the ensemble.

With the old mechanics all this involved ideas which for many readers were distinctly hard to accept. The principle of probability, embodied in the averaging over the ensemble, was frankly laid on top of the logical principles of Newtonian mechanics, and to anyone believing that probability would ultimately be brought down to the old logic the association was most repellent. But we can now see that Gibbs was a prophet far ahead of his time-and indeed, to be frank, far ahead of his own knowledge-for the new mechanics accommodates the ensemble very much more easily than did the old. The new mechanics has shown us that it is impossible to know how the individual molecules are moving, because when one undertakes an experiment to see, that experiment automatically alters the condition of the gas and so fails to tell what was wanted, the state of the molecules without the experiment.

In the old days one used to feel that the validity of Gibbs's idea would be spoilt by some skilful experimenter who would really observe the motions of the individual molecules and would therefore rule out the legitimacy of averaging over the whole ensemble, but we now know that there is no danger of this. The real gas in the vessel is not merely one specimen of the ensemble, unrecognizable only because of our clumsiness; it is itself the whole of the ensemble. We used to think of the gas as either in the state $A$,or in the state $B$, or in $C$, but according to the new physics we have to think of it as in all the states $A$ and $B$ and $C$. The distinction is typical of the change we must make in our habits of thought, and most of us resist this change strongly, for we find we can scarcely help asking: 'But which state was it really in ?' As I have said, we used to be ashamed of ignorance, but we must now realize that this ignorance is one of the things that makes the world possible. The principle of probability, which used to be loosely superposed on the old logical principle, is now with the new mechanics fully united with it in a higher synthesis.
Before leaving Gibbs I would like to refer to one thing in his book, where I think he has not even yet come into his own. He considers various types of ensemble of increasing generality. In the micro-canonical the members all have the same energy. Now we never know the exact energy of the gas in a vessel, so that a better idea is the wider one of a gas at a given temperature which therefore has a certain range of admissible energies. This is represented by Gibbs's canonical ensemble, and it is the main one that he uses. In both these the number of atoms in the ensemble is constant. But in the last chapter of his book Gibbs introduces a still wider ensemble. He calls the ones with a constant number of atoms petits ensembes, which I shall translate as petty ensembles, and regards them as parts of a grand ensemble in which the total number of atoms is not fixed. He uses the idea to some extent in connexion with semipermeable membranes, but on the whole does not get far with it.

As in much of Gibbs's work, it is the idea itself, rather than what he does with it, that is important. This idea of the grand ensemble is not yet incorporated in the new physics. In the quantum theory we take a number of electrons and nuclei, and, allowing for their interactions, we construct something that is practically the canonical ensemble. But we take fixed numbers of them-this is partly reflected in the technical process of using normalized wave-functions. Now in an experiment dealing with a large number of particles we are never really sure exactly how many there are, and to assume this number is much like assuming a constant energy for them. If the canonical ensemble is a better idea than the microcanonical, then the grand ensemble is superior to the petty ensemble. In the new mechanics nobody has yet succeeded in making anything of it, or has made any proposal how to do so, but I. will venture the forecast that when some of our present difficulties in the quantum theory are cleared up, it will be found that we shall be using the grand ensemble with its indefinite number of atoms.

Reverting to my main theme, what is the moral of all this? It is that the new physics has definitely shown that Nature has no sharp edges, and if there is a slight fuzziness inherent in absolutely all the facts of the world, then we must be wrong if we attempt to draw a picture in hard outline. In the old days it looked as if the world had hard outlines, and the old logic was the appropriate machinery for its discussion. Things went wrong when it was found necessary to call in the help of the principle of probability ; this appeared first as an alien, but there was hope in the old days that the alien might be naturalized. It has 
resisted the process and we now recognize that it cannot be assimilated, because it provides the necessary step to a wider reason, that of the new fuzzy world of the quantum theory, a world which is not contained in the old. How far it will be possible to make a full synthesis of the new and the old I do not know, but I like to think there is something in my analogy from the history of the quantum theory, and to suppose that we are still in the condition corresponding to the Old Quantum Theory, and that some day a real synthesis will be made like that of the New Quantum Theory, so that there will be only one thing in the world that has not indefinite outlines, and that will be a new reformed principle of reasoning.

There may be a feeling among some that the very general suggestions I have been making are open to every sort of criticism. Perhaps they are right; as I have said, it is part of my doctrine that the details of a physicist's philosophy do not matter much. But whether it is wrong or right, my next point is one on which $I$ do very much hope that there may be a consensus of agreement. This is that the subject of probability ought to play an enormously greater part in our mathematical-physical education. I do not merely mean that everyone should attend a course on the subject at the university, but that it should be made to permeate the whole of the mathematical and scientific teaching not only at the university but also at school. To the best of my recollection, in my own education I first met the subject of probability at about thirteen years of age in connexion with the problems of drawing black and white balls out of bags, and my next encounter was not until the age of twenty-three, when I read a book-I think it was on the advice of Rutherford-on the kinetic theory of gases. Things are better now, but mathematicians are still so interested in the study of rigorous proof, that all the emphasis goes against the study of probability.

This is not the place to describe a revised scheme of education. I would only say that it is not special new courses that are needed, but rather a change in the spirit of our old courses. When a boy learns about the weighing machine, emphasize its sensitivity, and consider the length of time that must be taken for the weighing. When he has a problem on projectiles, make him consider the zone of danger and not merely the point of fall. At a rather higher level, but still I should hope at school, introduce the idea of a distribution law ; for example, in doing central orbits work out Rutherford's law of scattering. Calculate the fluctuations of density of a gas, or the groupings in time of the scintillations of $\alpha$-particles. All these things ought to be examples of a familiar train of thought, and not merely a highly specialized side-branch of mathematics first met at the university. It is the incorporation of probability in the other subjects on which I want to insist, but there will of course remain some higher aspects-things like least squares or significance tests-which are still to be treated in separate university courses. Even these I should hope would come to be recognized as subjects of central interest and not, as they are at present, relegated to a remote corner of specialized study.

If these reforms are carried out. I shall hope that generations will grow up which have a facility that few of us at present possess in thinking about the world in the way which the quantum theory has shown to be the true one. The inaccuracies and uncertainties of the world will be recognized as one of its essential features. Inaccuracy in the world will not be associated with inaccuracy of thought, and the result will be not only a more sensible view about the things of ordinary life, but ultimately, as I hope, a fuller and better understanding of the basis of natural philosophy.

\section{Obituary Notices}

Sir John Snell, G.B.E.

$\mathrm{B}^{\mathrm{r}}$ $Y$ the death of Sir John Francis Cleverton Snell, the electrical profession has lost an inspiring leader, and all who knew him mourn the loss of a great-hearted and much-loved friend.

The son of Commander Snell, he was born in 1869, at Saltash, Cornwall, the county which produced Trevethick, Humphry Davy and other pioneers. Being unable to enter the Navy through defective eyesight, he decided to adopt an engineering career. He became a student of King's College, London (of which, in later years, he was elected a fellow), and after gaining valuable experience with Woodhouse and Rawson, and Crompton and Company (for whom he laid a complete system of underground mains in Stockholm), he became chief assistant to the late General Webber, and in 1893 became resident engineer to the Vestry of St. Pancras. From 1896 until 1906 he was borough electrical and tramways engineer of Sunderland, where he designed a new, and the main, power station and a complete system of electrical tramways.

In addition, Snell found time to publish in 1906 a work on the "Distribution of Electrical Energy", 